\title{
Rapid Stain Identification (RSID-TMSemen): A rapid tool for seminal fluid detection
}

\author{
Asmaa Abdel Rahman Abdel Rahman ${ }^{1}$, Sarah A. Khater ${ }^{2}$, and Eman Abd El-Hakim Attia ${ }^{1}$, \\ ${ }^{1}$ Forensic Medicine and Clinical Toxicology Department, Faculty of Medicine, Ain Shams University, Cairo, Egypt. \\ ${ }^{2}$ Forensic Medicine and Clinical Toxicology Department, Faculty of Medicine, Misr University for Science and Technology, \\ Cairo, Egypt.
}

\begin{abstract}
Introduction: The identification of semen stain is one of the most common human stains that can provide crucial information for crime scene reconstruction and forensic investigation. In sexual assault cases semen identification helps to support or refute victim's allegations, it also provides a material for DNA analysis. The rapid Stain Identification of Human Semen (RSID ${ }^{\mathrm{TM}}$-Semen) bioassay is designed to detect specifically the presence of human semenogelin. It does not crossreact with other human or nonhuman tissues.

Aim: To assess the efficacy of RSID ${ }^{\mathrm{TM}}$ - Semen strip test for the detection of human semen under some different variables (different fabrics, different time intervals and mixed with vaginal secretions).

Methodology: Semen samples were collected from four male participants; each sample was divided into two portions; one used for semen only test group and the other mixed with vaginal secretions for the mixed test group. Vaginal swabs were carried out from the four female participants using cotton, linen or nylon-tipped plastic rods (2 swabs from each female). One of the fabrics tipped vaginal swab was mixed with semen for the mixed test group and the other used as a positive control group to test the sensitivity and specificity of the RSID ${ }^{\mathrm{TM}}$ - Semen strip. The semen samples were deposited over different fabrics at the same time. All the samples were left to dry for 15 minutes at room temperature (summer) then extracted and analyzed. Each of the previous groups, was categorized into 5 subgroups (a, b, c, d and e) according to the time interval of semen extraction (zero (on the spot), 2, 4, 6 and 10 days respectively).

Results: Semen could be identified in 100\% of tested samples of the semen only group as well as of the combined semen and vaginal secretions group over cotton and linen fabrics at all the different tested time intervals. However, semen extracted from nylon fabric was identified in tested samples of the semen only group and of the combined semen and vaginal secretions group only at zero time only and couldn't be identified at the rest of tested time intervals.

Conclusion: The current study evidenced that the new $\mathrm{RSID}^{\mathrm{TM}}$-semen kit is a reliable method for semen identification over different types of fabrics even in the presence of vaginal secretions. It also persists up to 10 days except on nylon fabric.
\end{abstract}

Key words

Rapid stain identification test of semen - semenogelin - semen identification

\section{Introduction}

$\mathrm{B}$ ody fluid identification is an important component in forensic science, as the ability to identify body fluids, such as blood, semen, saliva and ....etc. is often the key in a criminal investigation and is subsequently relied upon in court. Many body fluid stains are invisible, present in very small quantities or mixtures, and so identification is not always easy. Identification tests for body fluid tests relied on chemical or enzymatic assays that were often presumptive in nature and generally limited in specificity or sensitivity. While confirmatory tests depend on microscopic or immunological tests, many of the early tests consumed the already limited biological material and were incompatible with DNA profiling which is a very crucial step in a wide range of investigations (Butler, 2012). 
As body fluids can be deposited on a variety of surfaces, tests also need to be able to work successfully on different substrates allowing subsequent analysis by DNA profiling (Harbison and Fleming, 2016).

Sexual assault is usually a hidden crime where the only witnesses are the victim and the assailant. In this violent crime, the assailant often leaves behind a personal biological signature including blood, saliva, and most importantly, semen. This semen evidence is a reliable marker in the investigation and in confirming the sexual assault. As the number of sexual assault cases are increasing day by day the need of detection of semen even if present in small quantity, no matter how old the stain is, should be detected precisely (Harel et al.,2015). So, one of the primary aims of the forensic laboratory in these type of cases is to sample and examine the biological material taken from the victim or stains found on cloths for the presence of semen,with the potential to link them with a suspect (Virkler and Lednev, 2009).

Biochemical techniques most recommended for routine forensic rape analysis include sperm cytology (SC), acid phosphatase activity (APA) and detection of prostate-specific antigen (PSA). SC is the gold standard confirmatory test; APA is a presumptive test, whereas PSA detection represents a more specific marker. But, false-positive reactions to urine, vaginal fluids, breast milk, and semen-free postmortem rectal swabs have been observed in methods that are currently used including immunological tests detecting PSA. Also, all these biomarkers have shown different stabilities in the vaginal fluid which may cause misinterpretation of results (Harbison and Fleming, 2016).

One of the emerging techniques in semen detection is commercial rapid stain identification (RSID)Semen Test. The RSID-Semen Test is designed to detect specifically human semenogelin (Sg) using monoclonal anti-human Sg antibodies. It involves the immunochromatographic membrane assay technology (Virkler and Lednev, 2009).

$\mathrm{RSID}^{\mathrm{TM}}$-Semen is designed for fast, easy, and reliable detection of human semen from a variety of samples encountered by forensic laboratories including clothing, bedding, vaginal swabs, and stained surfaces (Independent Forensics, 2016).

\section{Aim of the work:}

To assess the efficacy of RSIDTM - Semen strip test for the detection of human semen under some different variables (different fabrics, different time intervals and mixing with vaginal secretions).

\section{Materials and Methods}

Materials:

A) Materials used for semen extraction and identification:

Rapid Stain Identification of Human Semen Kit $\left(\right.$ RSID $^{\mathrm{TM}}$

- Semen). Cat NO. /ID: 0200. Manufactured by:

Independent Forensics. Kit contains:

1) Test cassettes: 25 cassettes individually wrapped and sealed in a moisture proof foil (a silica gel desiccant pouch has been added).

2) $5 \mathrm{ml}$ of RSID ${ }^{\mathrm{TM}}$ - Semen Running Buffer.

3) $25 \mathrm{ml}$ of RSID ${ }^{\mathrm{TM}}$-Semen Running Extraction Buffer.

B) Tested surfaces:

1- Fabric made of cotton $10 \mathrm{~mm}^{2}$.

2- Fabric made of linen $10 \mathrm{~mm}^{2}$.

3- Fabric made of nylon $10 \mathrm{~mm}^{2}$.

$>$ Ethical consideration:

A written informed consent were taken from each volunteer before participating in this study, and clarifying the aim of the study, the type of the required sampling and the analyses that will be done on samples donated in addition to scientific benefits to be expected from the application of the community.

All participants were assured about the confidentiality of all data, the finding discovered during examination and preservation of the samples. In addition, the right to refuse participation in the study was confirmed for all participants before obtaining consent for participation in this study. Also, no physical, moral, social or health hazards were inflicted on the participants in this study. After that, the approval of the ethical committee at Faculty of Medicine Ain Shams University (Code number: FWA000017585) was obtained.

$>$ Methods of sample collection: Semen:

The study was carried out at the Department of Dermatology and Andrology, at Ain Shams University hospitals from males attending the fertility clinic of the hospital. These men were referred for semen analysis to the lab as part of male infertility workup. Detailed instructions were given before the collection of samples. These included abstinence from coitus for 3-4 days; samples were collected aseptically by masturbation into sterile wide-mouthed bottles according to (Sikka et al., 2015) .

Vaginal secretions:

- The study was carried out at the Department of Obstetrics and Gynecology, at Ain Shams University hospitals from females attending the fertility clinic of the hospital.

- The subject must refrain from any kind of sexual activity, douching, and inserting any intravaginal products for at least 48 hours prior to the collection of vaginal specimens. As well as it must be free from any menstrual blood. 
- Vaginal specimens were collected on cotton, linen and nylon-tipped swabs. The swab is inserted and rotated 360 degrees in all four quadrants of the vaginal vault.

- Two swabs of each fabric were collected from each female participant.

Grouping:

Samples were collected from eight adult participants, four males and four females.

Part of the semen samples was deposited directly over the cotton, linen and nylon and the other part of the samples was mixed with part of the vaginal secretions samples collected from female participants on cotton, linen or nylon-tipped swabs simulating sexual assault swabs.

The other part of the vaginal secretions samples was used as positive control group to test the sensitivity and positivity of the RSID ${ }^{\mathrm{TM}}$ - Semen strip.

All the samples of the different groups were left to dry for 15 minutes at room temperature then extracted and analyzed according to the protocol designed for the Rapid Stain Identification Test (RSID ${ }^{\mathrm{TM}}$ - Semen). The collected samples were studied as 2 test groups and one control group as follows:

Test groups:

Group (I): Semen only group:

Samples were collected from male participants in sterile sealed plastic cups and deposited over different fabrics (cotton, linen and nylon).

Group (II): Semen mixed with vaginal secretions group:

Vaginal tipped swabs were soaked in $50 \mu \mathrm{l}$ semen.

In each of the previous groups, samples were categorized into 5 subgroups (a, b, c, d and e) according to the time interval of semen extraction (zero (on the spot), 2, 4, 6 and 10 days respectively).

Control groups (III):

- Subgroup III (a): positive control group: Vaginal secretion only:

This group was designed to test the sensitivity and the specificity of the tested strips, at time interval (zero, 2, 4, 6 and 10 days).

- $\quad$ Subgroup III (b): negative control group:

RSID $^{\mathrm{TM}}$ - Semen kit's extraction and running buffer-only-were directly subjected to semen extraction procedure. This group was designed to detect the efficacy of the (RSID ${ }^{\mathrm{TM}}$ - Semen) kit and to determine that the extraction method was totally according to the standard operating procedure (described by the manufacturers).

Scoring results:

RSID $^{\mathrm{TM}}$ - Semen should be evaluated exactly 10 minutes after the addition of samples. (Fig. 1) illustrates expected results:
* A visible red line at the Control (C) position only, indicates a negative result. No Semenogelin detected but the strip test is working correctly

* Visible red lines at both the Control (C) and Test (T) positions indicate a positive result. Semenogelin detected.

A visible red line at the Test $(\mathrm{T})$ position only indicates a failed test. Test failure, no possible conclusion.

\section{Results}

Results of semen extracted from different fabrics(Cotton, Linen and Nylon fabrics) and identified by RSID ${ }^{\mathrm{TM}}$ Semen method at different time intervals were as follows:

Samples extracted from the cotton and linen fabrics: $100 \%$ of tested samples of the semen only group as well as of the combined semen and vaginal secretions group gave positive results (semenogelin was detected) over these fabrics at different tested time intervals (0, 2, 4,6 and 10 days).This was easily determined by visual inspection of red lines at both the control (C) and test (T) positions of the strip (table (1) and figures 1 and 2). On the other hand, $100 \%$ of samples extracted from the vaginal secretions only group over the previous fabrics at the previous time intervals gave negative results (no semenogelin detected). This was easily determined by visual inspection of red line at the control (C) position only of the strip (table (1) and figures 1 and 2).

$>$ Samples extracted from the Nylon fabrics:

$100 \%$ of tested samples of the semen only group as well as of the combined semen and vagina group gave positive results (semenogelin was detected) over this fabric at 0 day only. This was detected by inspection of red lines at both the control (C) and test ( $\mathrm{T}$ ) positions of the strip.While samples extracted from the previous groups at other tested time intervals (2, 4, 6 and 10 days) gave negative results, red line at the control (C) position only of the strip. Furthermore $100 \%$ of samples extracted from the vaginal secretions only group over the nylon fabric at all the tested time intervals gave negative results (no semenogelin detected), (table (1) and figures 1 and 2).

The negative control samples (extraction and buffer solutions) of the (RSID ${ }^{\mathrm{TM}}$-Semen) gave a visible red line at the control (C) position only that indicates a negative result (absence of semenogelin)(table (1) and figures 1 and 2). 
Table (1): Shows results of semen identification extracted from different fabrics(Cotton, Linen and Nylon fabrics) versus the control groups; analysed by RSIDTM-Semen method at different time intervals (0, 2, 4, 6 and 10 days). Number of participants $=8$.

\begin{tabular}{|c|c|c|c|c|c|c|c|c|c|c|c|c|c|c|c|}
\hline Samples & \multicolumn{5}{|c|}{ Semen only } & \multicolumn{5}{|c|}{$\begin{array}{l}\text { Semen and Vaginal } \\
\text { secretions }\end{array}$} & \multicolumn{5}{|c|}{ Vaginal secretions only } \\
\hline Time intervals & $\begin{array}{c}0 \\
\text { day }\end{array}$ & \begin{tabular}{|c|}
2 \\
Days \\
\end{tabular} & \begin{tabular}{|c|}
4 \\
days
\end{tabular} & $\begin{array}{c}6 \\
\text { days }\end{array}$ & $\begin{array}{c}10 \\
\text { days }\end{array}$ & $\begin{array}{c}0 \\
\text { day }\end{array}$ & \begin{tabular}{c|}
2 \\
Days
\end{tabular} & \begin{tabular}{|c|}
4 \\
days
\end{tabular} & $\begin{array}{c}6 \\
\text { days }\end{array}$ & $\begin{array}{c}10 \\
\text { days }\end{array}$ & $\begin{array}{c}0 \\
\text { day }\end{array}$ & $\begin{array}{c}2 \\
\text { days }\end{array}$ & \begin{tabular}{|c|}
4 \\
days
\end{tabular} & $\begin{array}{c}6 \\
\text { days }\end{array}$ & $\begin{array}{c}10 \\
\text { days }\end{array}$ \\
\hline Cotton fabric & + ve & + ve & + ve & + ve & + ve & + ve & + ve & $+\mathrm{ve}$ & $+\mathrm{ve}$ & $+\mathrm{ve}$ & - ve & - ve & - ve & - ve & - ve \\
\hline Lenin fabric & + ve & + ve & + ve & + ve & + ve & + ve & $+\mathrm{ve}$ & + ve & $+\mathrm{ve}$ & $+\mathrm{ve}$ & - ve & - ve & - ve & - ve & - ve \\
\hline Nylon fabric & + ve & - ve & - ve & - ve & - ve & + ve & - ve & - ve & - ve & - ve & - ve & - ve & - ve & - ve & - ve \\
\hline $\begin{array}{c}\text { Extraction and buffer } \\
\text { solutions (negative control) }\end{array}$ & & & & & & & & & & & & & & & \\
\hline
\end{tabular}

$+v e=$ semen was identified

-ve = semen can't be identified

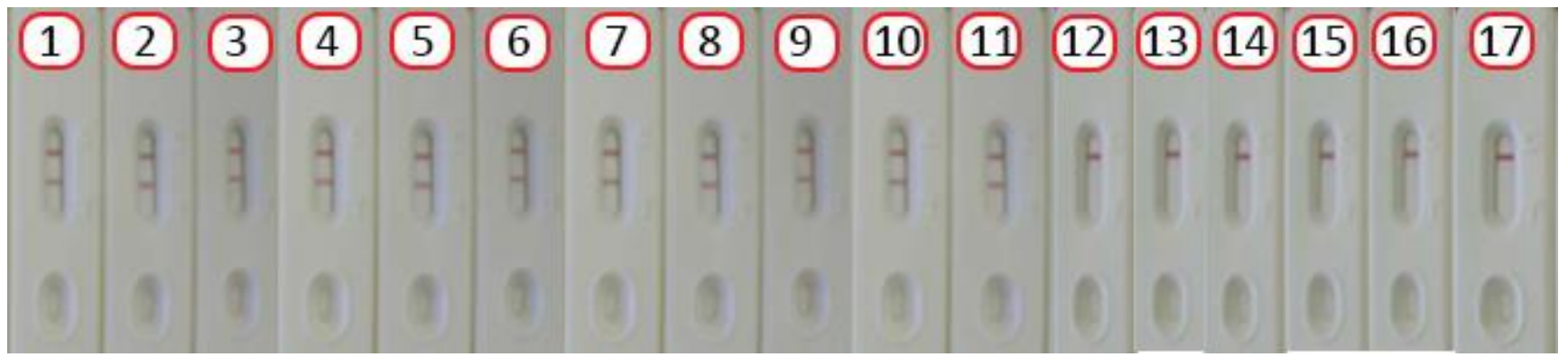

- Strips 1,2,3,4 and 5 represent semen only positive samples collected over cotton at 0,2,4,6 and 10 days respectively.

- Strips 6,7,8,9 and 10 represent positive semen only samples collected over linen at 0,2,4,6 and 10 days respectively.

- Strips 11 represents positive semen only samples collected over nylon fabric at 0 day and strips 12,13,14,15 represent negative semen only samples on nylon fabric at 2,4,6 and 10 days respectively.

- Strips 16 and 17 represent negative and positive controls respectively

Fig. (1): Photograph of the test cassettes for RSID ${ }^{\mathrm{TM}}$-Semen Kit showing results of identification of semen samples collected and extracted from different fabrics at different time intervals

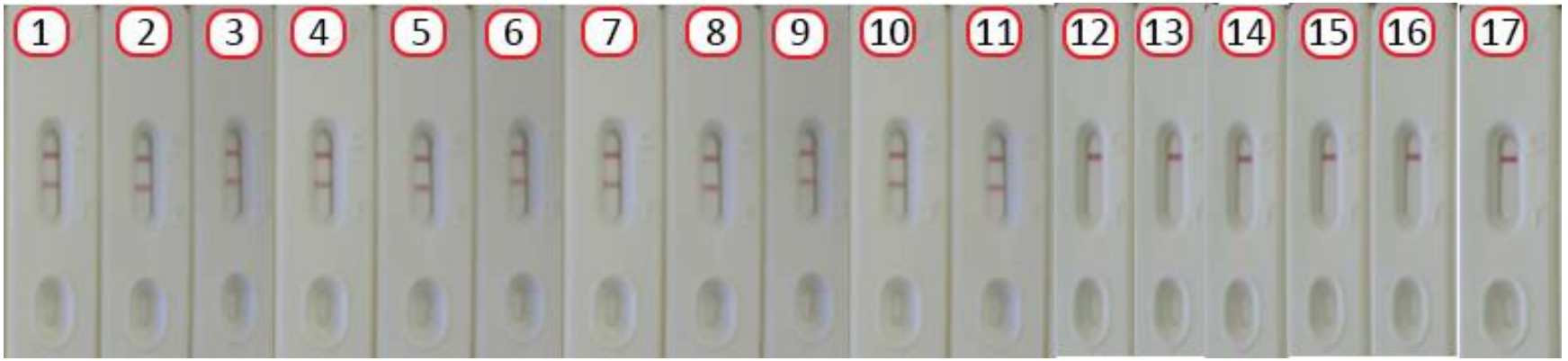

- Strips 1,2,3,4 and 5 represent mixed semen and vaginal positive samples collected over cotton fabric at 0,2,4,6 and 10 days respectively.

- Strips 6,7,8,9 and 10 represent positive mixed semen and vaginal positive samples collected over linen at 0,2,4,6 and 10 days respectively.

- Strips 11 represents positive mixed semen and vaginal positive samples collected over nylon fabric at 0 day and strips 12,13,14,15 represent negative mixed semen and vaginal positive samples on nylon fabric at 2,4,6 and 10 days respectively.

- Strips 16 and 17 represent negative and positive controls respectively.

Fig. (2): Photograph of the test cassettes for RSID ${ }^{\mathrm{TM}}$-Semen Kit showing results of identification of semen samples collected and extracted from different fabrics at different time intervals. 


\section{Discussion}

In forensic or police case work the identification of biological stains; blood, saliva, and semen is a daily task. Not only to know the origin of the body fluid that is important for the correct handling of the evidence in the laboratory, but also to reconstruct what may have occurred during the crime and to determine which items of evidence should be further processed for DNA analysis which is a very crucial step in a wide range of investigations (Old et al., 2012).

Sexual aggression constitutes a serious social and public health problem that calls for an urgent forensic medical examination (FME), particularly in acute cases; $\geq 72$ hours between the assault and the FME). Sexual assault cases are characterized by low rates of disclosure, reporting, prosecutionand conviction. Biological evidence is sometimes the only way to prove the occurrence of sexual contact and to identify the perpetrator (Magalhães et al., 2015).

Following sexual assault, swabs are usually taken from the victim, and, possiblyfrom the perpetrator. Most frequently swabs are taken from the external genitalia and the vaginal cavity, as well as from the mouth and anus. A second large group of evidence is secured from textiles; (underpants, bed sheets, blankets or jackets etc..) (Evers et al., 2009).

Semen is one of the most commonly encountered body fluids at crime scenes. However, sexual assault cases have varying factors that may mask semen findings when analyzing evidence at the forensic laboratory (Martínez et al., 2015).

In the absence of the microscopic identification of spermatozoa, semen was typically detected using the presumptive test for seminal acid phosphatase (an enzyme secreted by the prostate gland) but this is not specific to seminal fluid. The glycoprotein, PSA also known as P30 or kallikrein 3, is another biomarker that has been used to identify semen for some time (Old etal., 2012).PSA was also found in large amounts in seminal plasma; even after vasectomy as well as in other biological fluids, including female fluids and male urine. A potential limitation in using PSA to detect semen is its relatively rapid decay over time in vaginal fluid. So, PSA evaluation is not a sufficient method to detect seminal fluid in vaginal secretions 24-48 hours after coitus (Culhane et al., 2008). Semenogelin; a third potential biomarker for semen identification even in azoospermia cases or when few sperm are found. Other tissues including skeletal muscle, kidney, colon, trachea and lung tissues have also showed Sg, but significantly, for forensic purposes, no $\mathrm{Sg}$ is expressed in the female genital tract. This supports the use of Sg as a valuable method for semen detection compared to the PSA marker (Martínez et al., 2015).

Immunochromatographic lateral flow assay strip

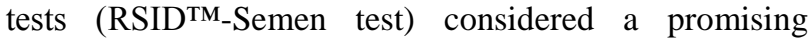
simple way to detect semen in a stain is aneasy, userindependent, quick, and cheap test. The kit was validated for forensic purposes by (Old et al., 2012). The idea of the test depends on the usage of two mouse monoclonal anti-bodies specific for human semenogelin, it is a confirmatory qualitative test for human semen that can detect as little as $2.5 \mathrm{~nL}$ of human semen and results are recorded as either positive or negative based on the presence or absence of a visible single red or blue line at the "Test" position on the strip 10 min following addition of semen to the sample well. The results are determined by visual inspection of the strip test and no image analysis or optical reader is required for scoring the test. There is no doubt regarding the results of the analysis; either the test band is visible or it is not. This detection protocol can be completely integrated into forensic laboratory procedures for DNA extraction, amplification before they are processed for DNA-STR analysis (Independent Forensics, 2016).

Despite the abundance of what was published on the effect of substrate materials on semen detection, however, these studies are limited to include certain materials as tile, concrete, wood, and cotton. There is a lack for research investigating other fabric types (Schlagetter and Glynn, 2017).

For the previous considerations, this research work was designed to simulate what real happen in crime scenes. Semen samples were collected from male participants by masturbation, part of the samples was deposited directly over the cotton, linen and nylon and the other part of the samples was mixed with vaginal secretions collected from female participants on cotton, linen and nylon-tipped swabs simulating sexual assault swabs. All samples were left to dry and to age at room temperature, and then each sample was subjected to semen extraction and identification by (RSID ${ }^{\mathrm{TM}}$-Semen) at its specific time interval.

In this study, the results showed that semen could be identified from semen only samples group deposited on both cotton and linen fabrics as well as from semen mixed with vaginal secretions group collected on cotton and linen swabs at different tested time intervals $(0,2,4$, 6 and 10 days). But semen was only identified on nylon fabric at 0 day but not at any of the other tested time intervals; (2, 4, 6, 10 days).

Pang and Cheung (2007)tested the degradation effect of vaginal fluid on two semen biomarkers (PSA and Sg) using twoimmunochromatographic identification methods (ABA card $^{\circledR}$ p30 and RSID ${ }^{\text {TM}}$-Semen kit) respectively. They found thatRSID ${ }^{\mathrm{TM}}$-Semen kit is more sensitive to detect Sg in semen samples extracted from vaginal fluid.

Hobbs et al. (2011)extracted semen from postcoital vaginal swabs using the same extraction method (buffered saline); for detection of two semen biomarkers (PSA and Sg) using ABA $\operatorname{card}^{\circledR}$ p30 and

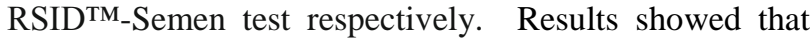
this method of extraction was compatible for ABA $\operatorname{card}^{\circledR} \mathrm{p} 30$. On the other hand, using this method for 
RSID ${ }^{\text {TM}}$-Semen kit hinder its sensitivity and gave negative results. They concluded that, to obtain reliable results for RSID ${ }^{\mathrm{TM}}$-Semen kit, manufacturer guidelines should be followed cautiously.

The results of the current study are in consistent with Old et al. (2012) who added semen to several subtypes of cotton fabrics as well as different subtypes of nylon and allowed them to air-dry completely before further processing. Each dried stain was sampled either by using distilled water (ddH2O)-moistened cotton swab or by excising a 5-mm diameter circle and dip it directly in the extraction buffer. Analysis was carried out according to the RSIDTM-Semen test protocol. Results revealed strong intensity band appeared $10 \mathrm{~min}$ at the test line for all fabric types sampled by the excision method. On the other hand, intensity of the bands of samples collected by the ddH2O-moistened cotton swab were type dependent; as they gave more intense signals with nylon compared to cotton fabrics. So, they concluded that using a swab tipped by the tested fabric itself gave reliable results than applying the ddH2O-moistened cotton swab.

This disparity in semen identification between different fabrics can be explained by the different absorbencies of the fabrics. The nylon did not readily absorb the biological fluids because it is a synthetic fabric that possesses more uniformity. Thus, it may not have retained as much semen stains as other fabrics, composed of natural fibers that easily absorb the biological fluids but had the stain retained on its outer surface. So, semen can be easily collected by the ddH2Omoistened cotton swab (Schlagetter and Glynn, 2017).

Old et al. (2012) analyzed in another stage of their previous study the detection of semen collected postcoital using cotton vaginal swabs at various time intervals, from individuals who had vaginal intercourse without the use of a condom. Analysis was performed daily from day 0 till day 10 then at day 14, 17 and 19. Results revealed that RSIDI ${ }^{\mathrm{TM}}$ - semen test could detect Semen in vaginal swab from day 0 up to day 2 only.

As opposed to the previous studies supporting RSID $^{\text {TM}}$-Semen test strips Boward and Wilson (2013) compared between ABA card and RSID ${ }^{\mathrm{TM}_{-}}$-Semen test strips for semen identification in postcoital samples. They stated that ABA card ${ }^{\circledR}$ p30detected the semen up to three days, while RSID ${ }^{\mathrm{TM}}$-Semen test showed very little sensitivity yielding a positive result only for the zerodaysample. They also criticized the RSID $^{\mathrm{TM}_{-}-S e m e n ~ K i t}$ as being more expensive and less reliable.

In a part of their study Laffan et al. (2011) studied the efficacy of RSID ${ }^{\mathrm{TM}}$-Semen testto identify semenfrom cotton fabrics after washing each fabric sample separately at $30 \mathrm{C}^{\circ}$ for 45 minutes and then left to dry for one hour. Semen was extracted using RSID ${ }^{\mathrm{TM}}$-Semen test strip where they gave positive results in all the tested samples. They concluded that semenogelinis a stable semen marker, resisting degradation when exposed to high temperatures. On the other hand, RSID ${ }^{\mathrm{TM}}$-Semen test is sensitive for semen detection despite its dilution through the washing process.

Martinez et al. (2015) chose RSID ${ }^{\mathrm{TM}}$-Semen kit for semen detection for its high sensitivity and specificity compared to other immunochromatographic tests targeting PSA as it is not specific; present in other body fluids such as female urine. Studies reported that the RSID $^{\mathrm{TM}}$-Semen test uses two mouse monoclonal antibodies specific for human semenogelin and can be used as a confirmatory test for human semen. It is sensitive; can detect the presence of semen down to $2.5 \mathrm{nl}$, accurate, easy to use, stable and non-detrimental analysis as the remaining extract can then be submitted for DNA analysis.

Holtkötter et al. (2018) found that RSID ${ }^{\mathrm{TM}}$-Semen kit gave positive results when identify ingmixtures of semen over shaded by the presence of human vaginal secretions.The study also demonstrated that semen samples alone or in mixtures containing saliva, semen, blood, menstrual fluid and urine left at room temperature; then extracted after 7, 14 and 21 days and identified by RSID ${ }^{\text {TM}}$-Semen kit gave positive bands; denoting test sensitivity.

Harbison and Fleming (2016) draw attention to the high-dose hook phenomenon, which occurs with all tests based on antigen-antibody reaction including RSID $^{\text {TM}}$-Semen test. They explained that weak positive results can be observed with large amount of analysed semen. This problem can be overcome by 10 -fold dilution of these samples and re-testing with RSID ${ }^{\mathrm{TM}}$ Semen which will eliminate the weak positive and false negative results.

\section{Conclusion and Recommendations}

In conclusion, the current study evidenced that the new RSIDTM-semen kit is a reliable method for semen identification over different types of fabrics and in the presence of vaginal secretions. It also persists up to 10 days.

Forensic researchers must address the issue of how best to perform semen detection when mixed with vaginal fluid.They must also study the effects of many variables (laundering, temperature, and whether the stain is dry or wet) on RSID semen sensitivity and specificity.

For the previous considerations the usage of RSIDTM Kits is highly recommended for semen identification as it is sensitive, specific, easy and persistent up to 10 days..

It is also recommended to quantify and analyze the condition of DNA extracted from semen using RSID $^{\mathrm{TM}}$-Semen for personal identification compared to the commonly used methods for qualification.

\section{References}

Boward, E. S., and Wilson, S. L. (2013): A comparison of

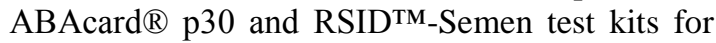
forensic semen identification. Journal of forensic and legal medicine, 20(8), 1126-1130. 
Butler J, (2012):Advanced Topics in Forensic DNA Typing: Methodology. Waltham, MA: Academic Press; 2012.

Culhane JF, Nyirjesy P, McCollum $K$ et al., (2008): Evaluation of semen detection in vaginal secretions: Comparison of four methods. American Journal of Reproductive Immunology, 60(3):274- 281.

Evers H, Heidorn F, Gruber C, et al,. (2009): Investigative strategy for the forensic detection of sperm traces. Forensic Sci. Pathol., (5):182-188.

Independent Forensics (2016): Rapid Stain Identification of Human Semen (RSID ${ }^{\text {TM}_{-}}$Semen), Technical Information \& Protocol Sheet for Use with Universal Buffer, Reduced Incubation Time Cat\#0230

Harbison S and Fleming R (2016): Forensic body fluid identification: state of the art. Research and Reports in Forensic Medical Science, 6:11-18.

Harel VS, Khairkar SR, Kulkarni KV et al., (2015): Detection of Semen Stains in Rape Cases by a Very High Powered UV-VIS Light Source , Facilitated Conviction of Accused Person. J. Forensic Res., 6(4): 4-6.

Hobbs MM, Steiner MJ, Rich KD et al., (2011): Vaginal swab specimen processing methods influence performance of rapid semen detection tests: A cautionary tale. J. Forensic Res., 82(3):291-295.

Holtkötter H, Schwender K, Wiegand P et al., (2018): Improving body fluid identification in forensic trace evidence-construction of an immunochromatographic test array to rapidly detect up to five body fluids simultaneously. Int. J. Legal Med. 132: 83-90.

Laffan, Á., Sawyer, I., Quinones, I., and Daniel, B. (2011): Evaluation of semen presumptive tests for use at crime scenes. Medicine, Science and the Law, 51(1), 11-17.

MagalhãesT, Dinis-Oliveira RJD, Silva B et al., (2015): Biological Evidence Management for DNA Analysis in Cases of Sexual Assault. Scientific world Journal, 1-11.

Martínez P, Santiago B, Alcalá, B et al., (2015): Science and Justice Semen searching when sperm is absent. Science \& Justice. Forensic Science Society, 55(2):118-123.

Old J, Schweers B, Boonlayangoor PW, et al., (2012): Developmental Validation Studies of RSID TM Semen A Lateral Flow Immunochromatographic Strip test for the Forensic Detection of Seminal Fluid Independent Forensics J. Forensic Sci. 54(4): 866-873.

Pang, BCM and Cheung, BKK(2007): Identification of human semenogelin in membrane strip test as an alternative method for the detection of semen. Forensic Science International169(1): 27-31.

Schlagetter T and GlynnCL (2017): The Effect of Fabric Type and Laundering Conditions on the Detection of Semen Stains. Int J of Forens Sci The Effect of Fabric Type and Laundering Conditions on the Detection of Semen Stains. International Journal of Forensic Sciences 2(2):1-8.

Sikka S, Baran C, and Hellstrom W (2015): A comparative evaluation of semen parameters in pre- and postHurricane Katrina human population. Asian Journal of Andrology17(4): 676.

Virkler K and Lednev IK (2009): Analysis of body fluids for forensic purposes : Laboratory testing to nondestructive rapid confirmatoryidentification at a crime scene. Forensic science international 188: $1-17$.

\section{التعرف السريع للبقع (RSID- ${ }^{\text {TM }}$ Semen): أداة سريعة للكثف علي السائل المنوي}

$$
\text { 'أسماء عبد الرهم عبد الرهمن' , و سارة عبد الخسن خاطر' , و إيمان عبدالحكيم عطية' }
$$

يعد التعرف علي بقع السائل المنوي واحد من أكثر الصبغات البشرية إنتشارا ـ والتي من الممكن أن توفر معلومات جوهرية لإعادة بناء مسرح الجريمة والتحقيقات في

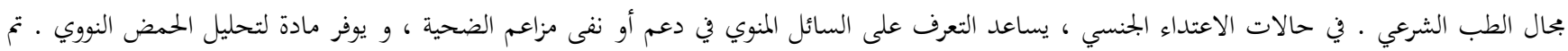

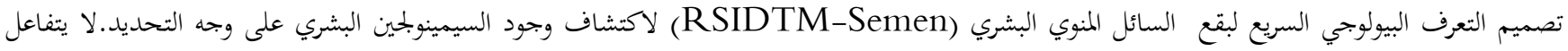
الاختبار مع الأنسجة البشرية أو غير البشرية الأخرى.

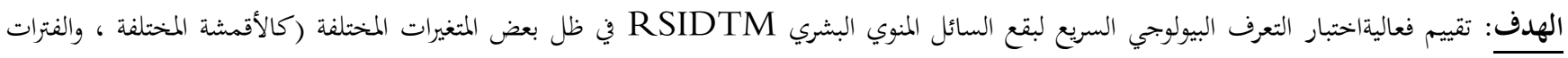
الزمنية المختلفة والبقع المختلطة بالإفرازات المهبلية).

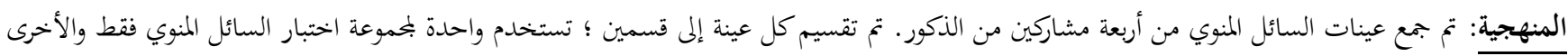

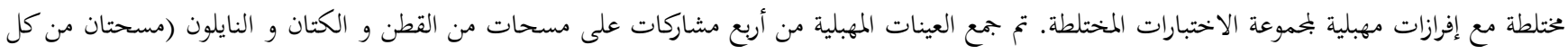


أنثى). تم خلط أحد الأقمشة ذات المسحة المهبلية مع السائل المنوي لبحموعة الاختبارات المختلطة والآخر استخدم كمجموعة ضابطة إيجابية لاختبار حساسية

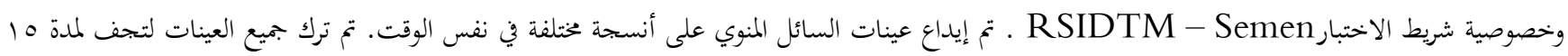

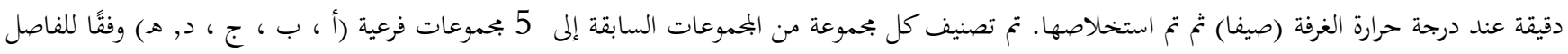

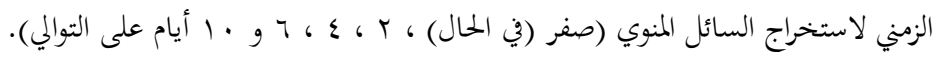

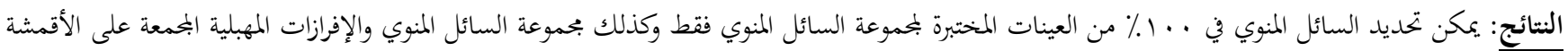

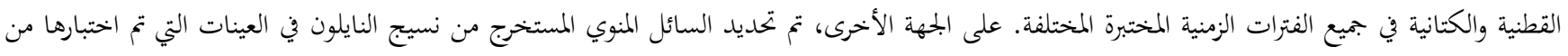

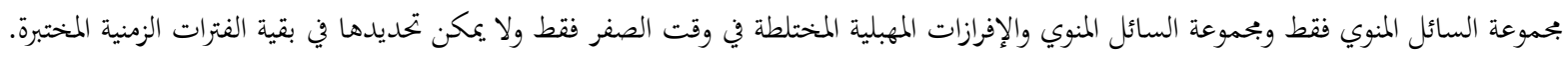

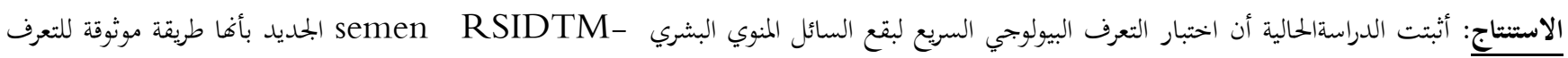

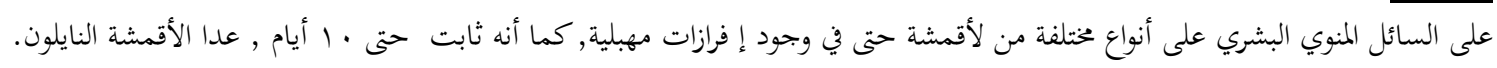

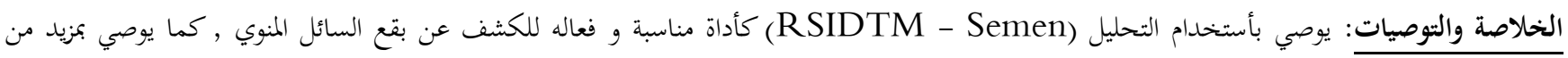
الأبحاث في هذا المحال وتطوير تحاليل أخري مناسبة. 